Kalganova T., G. Russell and A. Cumming (1999) Multiple Traffic Signal Control Using a GA. Proc. of the Fourth International Conference on Artificial Neural Networks and Genetic Algorithms (ICANNGA'99). Portoroz, Slovenia. Eds. Andrej Dobnikar, Nigel C. Steele, David W. Pearson, Rudolf F. Albrecht. Published by SpringerWienNewYork. pp. 220-228.

\title{
Multiple Traffic Signal Control Using A Genetic Algorithm
}

\author{
T. Kalganova, G.Russell, A. Cumming \\ Department of Computing, Napier University, Edinburgh, EH14 1DJ, UK \\ Email: \{t.kalganova, g.russell, andrew\}@dcs.napier.ac.uk
}

\begin{abstract}
Optimising traffic signal timings for a multiple-junction road network is a difficult but important problem. The essential difficulty of this problem is that the traffic signals need to coordinate their behaviours to achieve the common goal of optimising overall network delay. This paper discusses a novel approach towards the generation of optimal signalling strategies, based on the use of a genetic algorithm (GA). This GA optimises the set of signal timings for all junctions in network. The different efficient red and green times for all the signals are determined by genetic algorithm as well as the offset time for each junction. Previous attempts to do this rely on a fixed cycle time, whereas the algorithm described here attempts to optimise cycle time for each junction as well as proportion of green times. The fitness function is a measure of the overall delay of the network. The resulting optimised signalling strategies were compared against a well-known civil engineering technique, and conclusions drawn.
\end{abstract}

\section{Introduction}

One important and still difficult problem in traffic planning is the optimisation of traffic signals for a network of interconnected junctions, such that traffic moves through the network in an optimal way.

The main idea of traffic signal planning is that traffic signals in a road network can be co-ordinated so that the delay experienced by drivers is minimised over the duration of their journeys. By taking into account the available road-space at junctions and balancing the travel time between successive traffic signals, it is possible to derive widespread advantages in terms of free-flowing traffic and reduced overall journey time. Co-ordination between adjacent traffic signals involves designing a plan based on the occurrence and duration of individual signals and the time offsets (journey time between junctions). Sensor or survey information at junctions is also used to improve the overall timing plan of the network [1]. The research documented in this paper is concerned with the creation of optimum signal timing plans based on traffic survey information, with the fitness of the plan being calculated from the total delay incurred by the vehicles using the network. Delay is the additional time required to make a journey over and above that required to make the journey at normal driving speed without ever having to slow down.

There has been many studies carries out on the control of traffic signals $[2,3]$. Some of the approaches are based on the existence of a precise traffic model and research methods are applied to get the optimal control parameters. In such approaches a set of control parameters is created for each possible scenario envisaged in the network (e.g. a lane closed due to roadworks). The main disadvantage of this approach is that there are only a finite number of scenarios for which a traffic plan can exist, and if a scenario occurs for which no plan exists it is down to the operator of the traffic network signals to change the plan dynamically by hand to suit.

Other approaches are based on designing multi-agent systems in which the evaluation of each agent is carried out in simulation [2]. The first attempt to apply genetic programming and genetic algorithms (GAs) to traffic control is discussed in [3]. A fuzzy logic approach to network optimisation have also been examined [4,3], using heuristic rules as a way of adjusting signal timings.

Optimising a traffic network by optimising each junction in the network in turn does not usually result in a good solution, as each signal also should be optimised such that traffic flowing through a number of junctions in turn does not get stopped at each junction. This is important for arterial roadways through networks, where the objective is to allow vehicles to leave the network as quickly as possible. In addition, where sensor information is present at a junction, this may be utilized by other junctions in the surrounding area.

This paper presents a traffic control method based on GAs. The goal of the author's investigations was to generate near optimal traffic signal settings for each traffic controller in terms of minimal delay of an entire road network. The idea of this approach can be briefly described as follows:

1. Traffic signal timings are generated by a GA for the entire network.

2. A high-speed traffic simulator simulated the network using the traffic signal timings generated from the GA.

3. The simulator produced a fitness measure for the signal settings, and this was fed back into the GA. 
This list was iterated over until the GA was stopped, which occurred either when a execution-duration maximum or an apparent fitness maximum was reached (depending on the experiment being performed). The best timing diagrams generated by the GA for a particular 3 -junction network are given. These results are then compared with ones generated from theoretical analysis of the network.

\section{A Genetic Algorithm For Traffic Signal Control}

The objective in adjusting the timing of traffic control signals is to minimise the overall delay to traffic over an entire traffic network [5]. The GA described in this paper tackles the problem of obtaining good signal timings by evolving the signal proportions, offsets and the cycle time.

Each junction has a number of roads approaching it, and each approach gets some green time to allow cars into the junction. Consequentially, each approach also has red time during which cars on that approach must not enter the junction. The proportion of red time to green time for an approach is referred to as the signal proportion for that approach.

Each approach to a junction has a green period, and a red period. The total of the green and the red period is referred to as the cycle time.

Consider two junctions, one after another, along a particular route. Optimally, ignoring any other vehicles, a vehicle which was stopped at one junction and which then receives a green light should almost reach the second junction before receiving a green light at that junction, thus ensuring a delay-free journey. Thus it is useful to be able to describe when the next junction in a series goes green in comparison to the first junction. To perform this task, we use the idea of an offset time, which is basically the time between the whole network being switched on and approach 1 of the junction in question going green.

In the author's approach, signal timings for all the traffic controllers in the network are represented by a single chromosome. The fitness of the chromosome corresponds to the total delay for vehicles using the network over a fixed time period. The fitness is obtained from simulation, which uses random vehicle arrival rates into the edges of the network, constrained to meet a preset average vehicle flow. This allows us to develop optimal signal timing information which are robust for a variety of different traffic patterns.

\subsection{Traffic Signal Parameters}

Consider a road network containing $n$ junctions. Each junction controls its own signals using the following parameters:

- the inter-green period (IGP) - in a junction, when one light changes from green to red there must be a short delay before another signal in the junction can change from red to green. This is to give vehicles already in the junction a chance to leave. This is especially true for vehicles which are trying to turn across oncoming traffic, which may be trapped in the centre of a junction until the lights turn red. In Britain, IGP $=3$ seconds.

- the green and red time for a particular approach, $t_{g}$ and $t_{r}$ respectively;

- the cycle time for a particular junction, $t_{c}=t_{r}+t_{g}$;

- the offset time, $t_{o}$;

Signal timings are cyclic, in that they repeat themselves every $t_{c}$. It is useful to know how the separate junctions are synchronised with each other. The time $t_{o}$ gives an indication of this. Effectively, this is the time between activation of the junction signal controller and the time that the controller begins to follow the signal configuration. Specifically, the time $t_{o}$ is the difference between an absolute zero clock and the clock that turns the first of the signals in a junction to green. Each junction has an offset time. The traffic model is evaluated in the simulator for $t_{m}$ simulated seconds of traffic flow. For the experiments performed in this paper $t_{m}=960 \mathrm{~s}$. The graphical representation of some of these parameters are shown in Figure 1.

\subsection{Chromosome Representation}

Chromosome $C$ describes the behaviour of $n$ traffic controllers which is represented by $C$, where $C=<C_{1}, C_{2}$, $\ldots, C_{n}>$. Each $C_{i}=<b^{i} c_{1}^{i} c_{2}^{i} c_{3}^{i}>, i=1, \ldots, n$ defines all the parameters needed by the $i$-th controller. In our experiments we assume that people drive on the left-handside. A junction diagram can be found in figure 1 .

Signals are assumed to have only two colours: red and green. If $b^{i}$ is 0 , then when the junction is activated the light on the Main Street approach starts as red, and if it is 1 then the light starts at green. This is referred to as the first colour of the signal, and has a duration $\left(c_{1}^{i}+c_{3}^{i}\right) . c_{2}^{i}$ is the duration of the second colour, which is the opposite colour to that of the first colour. Based on this type of chromosome representation we can compute the cycle time for the $i$-th junction and offset time as follows:

$$
t_{o}=c_{1}^{i} \text { and } t_{c}=c_{1}^{i}+c_{2}^{i}+c_{3}^{i}+2 * \mathrm{IGP}
$$




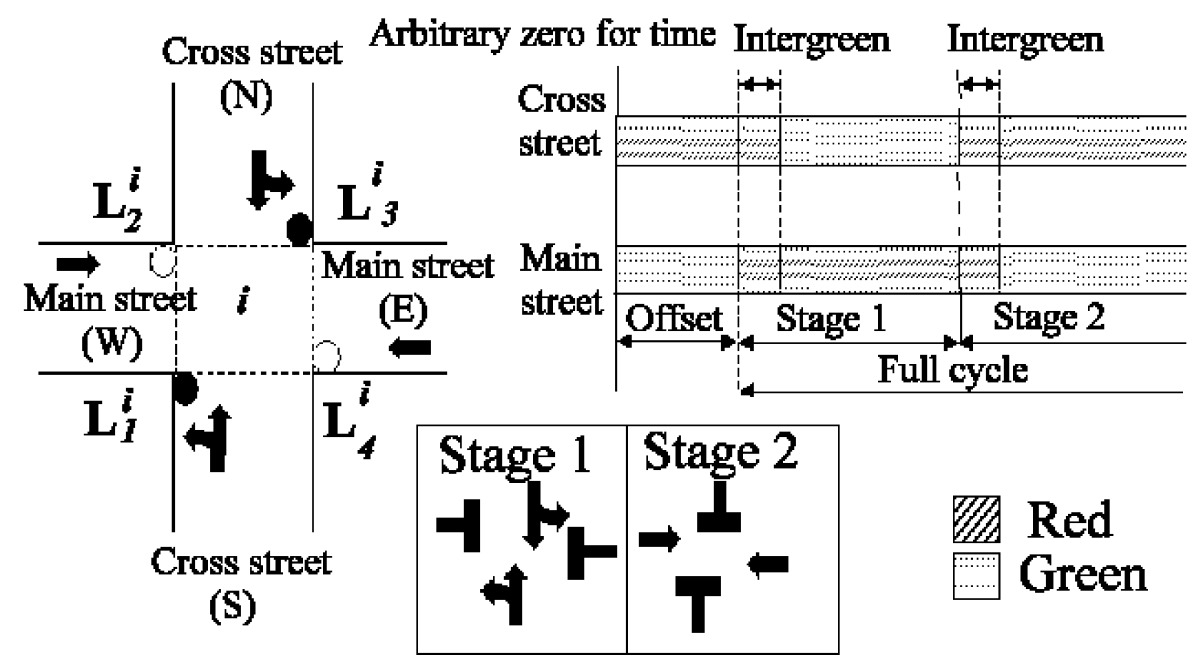

Fig. 1. Cycle time

If gene $b^{i}$ indicates that the first colour is green, then Main Street is:

$$
t_{g}=c_{1}^{i}+c_{3}^{i} \text { and } t_{r}=c_{2}^{i}+2 * \mathrm{IGP}
$$

otherwise:

$$
t_{r}=c_{1}^{i}+c_{3}^{i}+2 * \text { IGP and } t_{g}=c_{2}^{i}
$$

Cross Street $t_{g}$ is simply Main Street $t_{r}-2 *(I G P)$.

\subsection{Single-Junction Network Representation}

Consider the chromosome representation for the one-junction network shown in Figure 1. In this case chromosome $C=<C_{1}>$. By way of an example, assume $C_{1}=<\begin{array}{llll}0 & 15 & 25 & 10\end{array}$. First consider the traffic lights on Main Street. The traffic lights $L_{1}(i)$ and $L_{3}(i)$ will have the same signal. The start signal is red $(b=0)$, thus during the first $15 \mathrm{~s}\left(c_{1}=15\right)$ the traffic lights located on Main Street with be red. The IGP is $3 s$, so for the next $3 s$ the red signal is maintained. Then the signal is changed to green for $25 s\left(c_{2}=25\right)$. As the actual red time in this case is represented by the sum of the first and third genes, and take into account the double IGP (changing signals from red to green and then from green to red), the next red signal last for $31 s\left(c_{1}+c_{3}+2 *\right.$ IGP). The Cross Street signals are green IGP seconds after Main Street goes red, and remain green till IGP seconds before Main Street return to green. The cycle time is $56 \mathrm{~s}$. The timing diagram is shown in Figure 1.

\subsection{Multiple-Junction Network Representation}

Consider the three-junction road network shown in Figure 2. In this case the chromosome contains $<C_{1}, C_{2}, C_{3}>$, as the network has three junctions. An example of the chromosome representation for this network is given in Figure 2. The behaviour of each junction is described by a single $C_{i}$. Based on this the signal timings for each traffic light in every junction can be computed, and is shown in Figure 3. Note that in this representation each of the junctions can have a different cycle time and offset time.

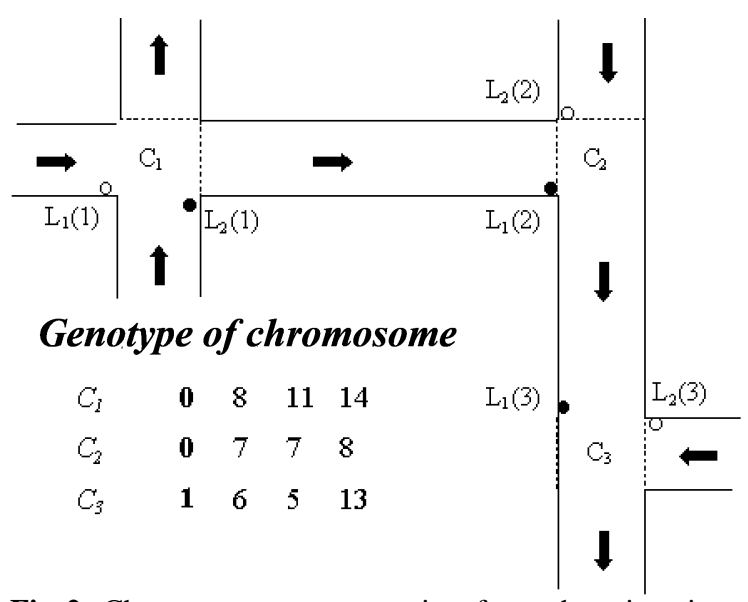

Fig. 2. Chromosome representation for a three-junction network 


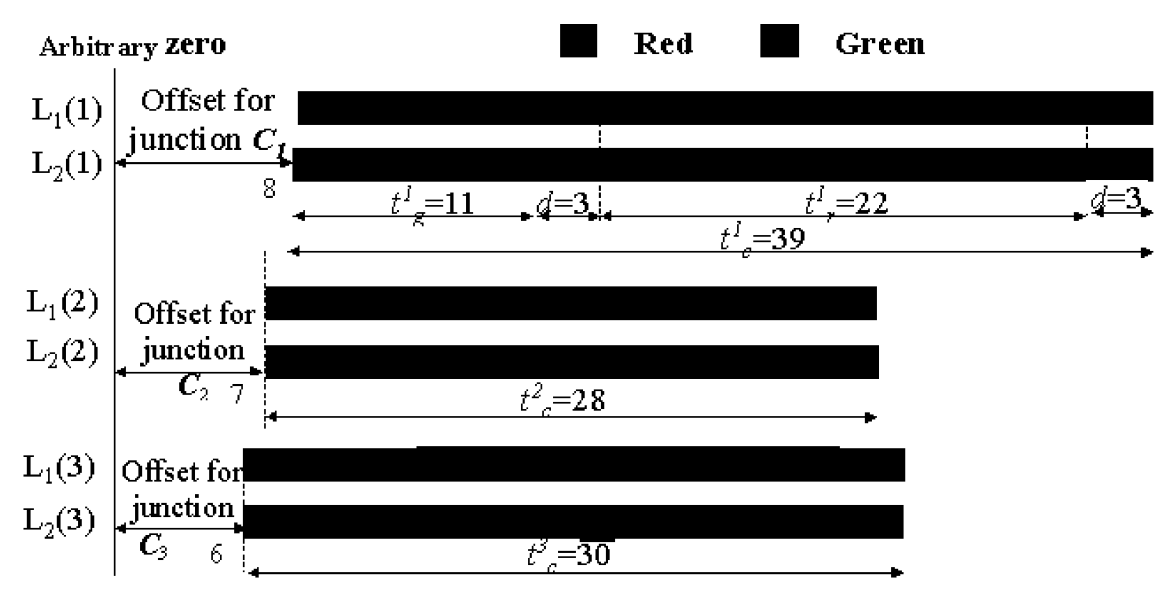

Fig. 3. Timing diagram for a three-junction network

\subsection{Preparation Of String Encoded Parameters}

A set of randomly initialised parameters $b^{i j}, c_{1}^{i j}, c_{2}^{i j}$ and $c_{3}^{i j}, i=1, \ldots, n ; j=1, \ldots, m$ is prepared such that $c_{1}^{i j}+c_{2}^{i j}+$ $c_{3}^{i j}+2 I G P \leq 130$, where $m$ is the number of chromosomes in population. In Britain all cycle times must lie between 20 and 130 seconds. The gene $b^{i j}$ defining the start signal for $L_{1}(i)$ traffic light is randomly initialised and takes value 0 or $1 . c_{k}^{i j}$ is randomly initialised in the range $[0,130]$, where $k=1,2,3$.

\subsection{Calculating Optimum Signal Settings}

The $j$-th chromosome $C(j)$ specifically the parameter set $\forall_{i} \forall_{k} c_{k}^{i j}$ is applied to the junction controllers $B_{i}, i=1$, $\ldots, n$ and the parameters used in a software simulation of duration $t_{m}$. The simulator returns the total delay incurred by vehicles travelling in the simulated network.

\subsection{The Genetic Search Operators}

Evolution of the population in the GA involves two basic steps: (1) a selection mechanism that implements a survival of the fittest strategy and (2) a genetic recombination of the selected chromosomes to produce offspring for the new population. Recombination is affected through the two genetic operators of crossover and mutation. A uniform mutation operator is used, where the mutated gene takes a randomly generated integer value from a specific range. In order to obtain the highest convergence within the GA different types of selection and crossover were investigated, as well as different sizes of population.

Two types of selection operators were used: roulette wheel and probabilistic binary tournament. With roulette wheel, probability of selection is proportional to fitness. With tournament selection, the probability of the tournament victor being selected is the tournament discriminator. Experimentation showed that the optimal tournament discriminator was 0.8 . This experimentation also indicated that tournament selection significantly outperformed

roulette wheel.

Three different types of crossover were implemented: (1) uniform, (2) one-point and (3) two-point. Cut points were chosen so that individual junctions were not perturbed. The best solution was obtained using uniform crossover, but the behaviour of mean fitness is better for two-point crossover. The convergence of the GA is faster for one-point crossover, and in the experiments discussed in the next section the one-point crossover operator is used. A number of experiments were carried out to discover the optimum mutation rate. The results of this indicate an optimum mutation rate of 0.03 . Using an adaptation technique for mutation rate, where the rate is increased when the fitness of the population reaches a local maxima, appears to improve the optimal solutions obtained during our experiments.

\section{Experimental Results}

In order to evaluate the usefulness of the GA in optimizing traffic signals, an experiment was constructed where the GA controlled the settings of the three simulated signalised junctions shown in Figure 2. Each of these junctions use a two phase signal configuration.

The experiment was constructed so that the GA selected a sequence of traffic signals, and then passed these to the simulation engine. Traffic was then simulated in 
the traffic network using the specified signal timings, and a measure of how well the traffic moved through the network was obtained. This measure was then returned to the GA as a fitness value, and the cycle repeated.

\subsection{The Simulated Road Network}

Ideally the GA would have been best connected to a realworld set of traffic signals, but the expense of this, not to mention the inconvenience to real road users as the GA tried to find an optimum signal setting means that getting permission to using this approach would have been difficult. Instead, we have used a urban traffic simulator, JUDGE [6], which was developed by the authors during research performed a few years ago at Strathclyde University. It makes use of probabilistic traffic arrival times, and simulates traffic using a microscopic discrete modelling technique $[7,8]$. With this system we can simulate a hour of traffic flow in a few seconds.

For the purposes of the evaluation, the JUDGE traffic simulator was programmed with the three-junction traffic network shown in Figure 2. JUDGE allows networks to be built up using a "train-set" approach, linking up roads with other constructs (such as junctions) by placing components next to each other on a two-dimensional surface.

The simple network used in the simulation is designed to be hard enough so that the solution is not easily solved without simulation, but is easy enough to understand what the GA has tried to achieve. The authors intend to look at more complicated networks with a higher traffic loading as a next stage in developing this research.

The GA produced traffic signalling information, which is then simulated by JUDGE for 16 minutes of traffic time (this takes about 1 second of CPU time). JUDGE also analyses the performance of the network, calculated vehicle delay, flows, average speeds and queue lengths. This information is then collated and formed into a fitness measure for the network. For simplicity, the fitness function is based solely on vehicle delay measures, although the use of the other measurements is also being investigated.

\subsection{Flows In The Network}

There is a number of possible settings for the signals in this network that produce optimal fitness measurements. No fitness should ever reach zero, as even on a straight roadway cars will interfere with each other (e.g. a fast vehicle may be slowed down by a slower- moving vehicle, causing delays).

The input flows for the network is shown in table 1. In traffic modelling, flows are measured in $\mathrm{pcu} / \mathrm{hr}$. A pcu, or Passenger Car Unit is a number which describes how big a vehicle is. For instance, a bicycle is $0.3 \mathrm{pcu}$, whereas the standard household vehicle is $1.0 \mathrm{pcu}$.

There are also turning probabilities for each approach road to junction 1, indicating how much of each traffic flow goes down each of the two roads which leave junction 1 . The result is that $700 \mathrm{pcu} / \mathrm{hr}$ travel from junction 1 to junction 2. The flow from junction 2 to junction 3 should be around $1000 \mathrm{pcu} / \mathrm{hr}$, and the output from junction 3 is around $1300 \mathrm{pcu} / \mathrm{hr}$. All roadways have a capacity of $1800 \mathrm{pcu} / \mathrm{hr}$, and a speed limit of $46 \mathrm{~km} / \mathrm{hr}$.

\subsection{Expected Results}

In civil engineering, the design of traffic signaling strategies can often be thought of as an art rather than a science. There are a large number of theoretical models which describe ways to calculate how good a particular signal strategy actually is. None of the theoretical models are recognised as being an accurate model of real-world traffic. Even the best software simulations of traffic flow must be, by definition, be a simulation of some of the important factors of real-world traffic networks. Thus, it is an almost impossible task to confirm that the results generated by the GA are in fact good, near-optimum results.

In order to give some confidence to the results produced by the GA, two separate analyses of the data was performed. Firstly and most simply, a study was made as to the proportion of traffic flows which arrive at a particular junction. This should give an indication as the the proportion of the green times which each approach should expect. A table depicting this information for the network used in analysing the GA can be found in table 1 .

Secondly, a respected theoretical model for steadystate single junction networks, Webster [9], was used to get a better understanding of the cycle times and green times produced by the GA. This theoretical model is based on queuing theory, and takes no account of the varying distribution of vehicles as they approach the junction (which happens in real-life traffic). Optimum signal settings for the network, as calculated from the Webster equation is also shown in table 1:

Note that neither of these two data analysis techniques take into account the fact that the junctions are combined together in a single network, but instead treat them all as separate entities. This is unfortunate, as related junctions will have to make compromises in their configuration in order to achieve a global optimal setting for the network. Nevertheless, it should give some sort of indication as to how well the GA has performed. 


\begin{tabular}{|c|c|c|c|c|c|c|}
\hline Junction & Approach Direction & Flow & Total & $\begin{array}{c}\text { Green Proportions } \\
\sigma_{g}^{\text {predicted }} \\
\end{array}$ & $\begin{array}{r}\text { Webster's } \\
\text { Green Time }\end{array}$ & $\begin{array}{l}\text { Predicted } \\
\text { Cycle Time }\end{array}$ \\
\hline Junction 1 & West & $\overline{500}$ & 1400 & $35 \%$ & 21 & 62 \\
\hline Junction 1 & South & 900 & 1400 & $65 \%$ & 35 & 62 \\
\hline Junction 2 & North & 300 & 1000 & $30 \%$ & 9 & 31 \\
\hline Junction 2 & West & 700 & 1000 & $70 \%$ & 16 & 31 \\
\hline Junction 3 & North & 1000 & 1300 & $76 \%$ & 31 & 49 \\
\hline Junction 3 & East & 300 & 1300 & $24 \%$ & 12 & 49 \\
\hline
\end{tabular}

Table 1. Green time proportions and Webster Predictions

\subsection{Obtained Results}

Table 2 contain the data analysis of several best signal timings defined by the genetic algorithm described above. The proportion of green time is defined as $\sigma_{g}$ and computed as follows:

$$
\sigma_{g}=\frac{t_{g}}{t_{c}-2 * d} * 100 \% .
$$

The proportion of green time for each traffic light in the three-junction network is given in Table 2 for different signal timing diagrams. The difference between the expected proportion of green time and that obtained by experimentation, $\Delta_{p}$, is calculated as follows:

$$
\Delta_{p}=\sigma_{g}^{\text {predicted }}-\sigma_{g}^{\text {obtained }} .
$$

When $\Delta_{p}$ is minimal over all traffic lights of network then the best fitness is obtained. It is interesting to note that the biggest differences are mostly found in the second junction, and that in the majority of cases the cycle time for this junction is also smaller than for the others. The best fitness (54.24) had been obtained with a population of 80 chromosomes, a genetic algorithm with tournament selection, and using a one-point crossover and uniform mutation. The mutation rate was 0.3 and crossover rate was 0.25 . Elitism was used to improve the GA convergence.

The table also shows $\Delta_{w}$, which it the difference between the $\sigma_{g}^{\text {obtained }}$ and that predicted by the Webster equation.

The analysis of these results indicates that the GA is excellent in finding near-optimum $\sigma_{g}$ values, as the corresponding $\Delta_{p}$ and $\Delta_{w}$ values equate to only about +/1 second in terms of the cycle times involved. This is to be expected, as neither the proportional study nor the Webster equation is as accurate as the software simulation.

What is interesting is that the optimum cycle times produced by the GA are for the most part all much lower than that predicted by Webster equation. Post analysis has given three main reasons for this; the fitness of a junction is governed more by green proportions than cycle time, the equations used treat each junction separately but yet in real-life junction settings must be changed due to the effect of surrounding junctions, and that the genome encoding used cannot modify cycle times without changing the green time proportions for a junction.

\subsection{Fitness Variations}

Two graphs have been created in order to demonstrate why the fitness measure was dominated by green time proportions rather than cycle times. They have been generated from the Webster equation.

In Figure 4, graph $b$ shows the effect of varying the green time for a junction (in this case Junction 2), while keeping the cycle time fixed, results in an obvious "saddle" area where the delays are quite similar in value. In this area, a green time of between 6 and 10 would produce similar delays. However, moving outside this area in either direction and the delay increases dramatically.

Figure 4, graph a shows the effect of varying the cycle time for a junction (this time Junction 3), while keeping the green time proportions fixed. There is a obvious "knee" area in the graph. In this area, the minimum delay is to be found, but it is significantly effected by noise. This is due to rounding effects causes by a simulation constraint that signal timings must be rounded to the nearest second. To the left of this area, the delay increases significantly, and to the right the delay increases more gradually.

The genome in the GA was such that the cycle time was calculated from the red and green time components of the genome. In this way the cycle time was inseparable from the green proportions. Increasing or decreasing the cycle time could only be achieved by changing the green proportions in the genome. As there is only a narrow area where green proportions had similar delay measures, and outside this area delay increased dramatically, 


\begin{tabular}{|c|c|c|c|c|c|c|c|c|c|c|c|c|c|}
\hline Junction & $\begin{array}{l}\sigma_{g} \\
\% \\
\end{array}$ & $\begin{array}{c}\Delta_{p} \\
\% \\
\end{array}$ & \begin{tabular}{|c|}
$\Delta_{w}$ \\
$\%$ \\
\end{tabular} & \begin{tabular}{|c|}
$t_{c}$ \\
$s$ \\
\end{tabular} & $t_{c}$ & $\begin{array}{l}\sigma_{g} \\
\% \\
\end{array}$ & $\begin{array}{c}\Delta_{p} \\
\%\end{array}$ & \begin{tabular}{|c|}
$\Delta_{w}$ \\
$\%$
\end{tabular} & $t_{c}$ & $t_{o}$ & $\begin{array}{l}\sigma_{g} \\
\% \\
\end{array}$ & \begin{tabular}{|c|c|}
$\Delta_{p}$ & $\Delta_{w}$ \\
$\%$ & $\%$ \\
\end{tabular} & \begin{tabular}{c|c}
$t_{c}$ & $t_{c}$ \\
$s$ & $s$ \\
\end{tabular} \\
\hline & $\overline{33.3}$ & $\overline{1.7}$ & 4.2 & 39 & $\overline{8}$ & 34.5 & $\overline{-0.5}$ & $\overline{3.0}$ & 35 & $\overline{8}$ & $\overline{33.33}$ & \begin{tabular}{|l|l|}
1.7 & 4.2 \\
\end{tabular} & \\
\hline uth & 66.6 & 1.7 & -4.1 & & & 65.5 & 0.5 & -3.0 & & & 66.64 & $-1.7-4.1$ & \\
\hline$C_{2}$, North & 31.8 & -1.8 & 4.2 & 28 & 7 & 41.2 & -11.2 & -5.2 & 40 & 8 & 31.6 & \begin{tabular}{|l|l|}
1.6 & 4.4 \\
\end{tabular} & 25 \\
\hline$C_{2}$, West & 68.2 & 1.8 & -4.2 & & & 58.8 & 11.2 & 5.2 & & & 68.4 & $1.6-4.4$ & \\
\hline$C_{3}$, North & 79.2 & -3.2 & $\mid-8.1$ & 30 & 6 & 73.5 & 2.5 & -2.4 & 40 & 12 & $\overline{75}$ & \begin{tabular}{|l|l|}
1.0 & -3.9 \\
\end{tabular} & 30 \\
\hline$C_{3}$, East & 20.8 & 3.2 & 8.1 & & & 26.5 & -2.5 & 2.4 & & & 25 & \begin{tabular}{l|l}
-1.0 & 3.9 \\
\end{tabular} & \\
\hline thess & & \multicolumn{5}{|c|}{60.3319} & \multicolumn{3}{|c|}{54.24} \\
\hline
\end{tabular}

Table 2. The best traffic signal control results obtained by GA

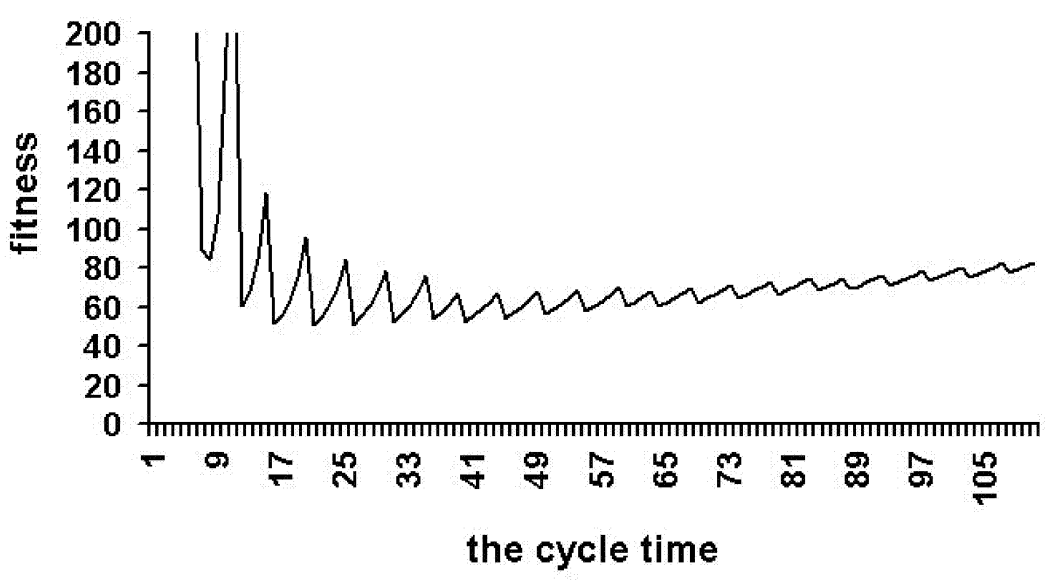

(a)

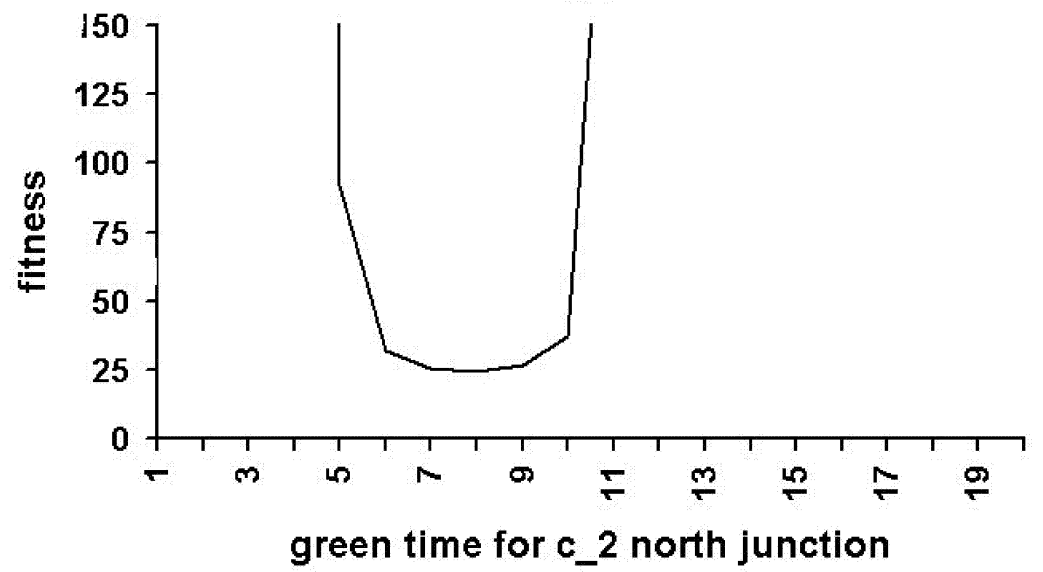

(b)

Fig. 4. Effect of varying cycle time while keeping green time proportions fixed 
attempts to increase the cycle time (and thus change the green proportions) would initially be given low fitness values, and would thus be considered as highly undesirable members of the population. In addition, as the change in delay caused by changes in cycle time are small in comparison to the effect of changes in green proportions, even a genome designed so that cycle time was independent of green proportions may mean that a GA may not have spent enough effort in producing an optimum cycle time.

From this analysis it is clear that, as a next step, cycle time should be isolated from the green proportions in some way, perhaps encoding it as a percentage green, percentage red, and a cycle time. However, some way to promote the breeding of better cycle times rather than concentrating on green proportions must also be developed.

\section{Conclusion}

Our novel approach to signal optimisation allow us to configure traffic controllers for a variety of geometric configurations and traffic conditions, by adapting the signal parameters of each traffic junction controller in terms of overall network delay. These parameters include cycle times, offsets, and green proportions. Our approach can be easily extended for different types of junctions and roadways. This is one of the future directions for our investigations.

The actual signal configurations produced by the GA were similar to that expected by the Webster equation; a traditional theoretical model for junction optimisation. Where the GA configured signals differed from Webster the cause can be traced to the way the chromosome was encoded, and to the fact that the Webster equation is based on a number of assumptions which are not present is either real traffic flows or the JUDGE simulator-based traffic model.

The GA investigation showed that the choice of genetic operators and their parameters has a marked effect upon GA performance, and that the choice is dependent on the GA components used. For the given chromosome representation the tournament selection operator performed better in comparison to the uniform operator. It was discovered that the best GA convergence was achieved when the tournament discriminator equalled $100 \%$. However, the best fitness result can be achieved for a tournament discriminator equalling $80 \%$.

Many key questions remain unanswered. These include: (1) how using multi-agent techniques instead of traditional genetic algorithm will influence the obtained result, (2) how our approach handles a larger number of junctions, (3) how our approach compares in performance terms with other adaptive control approaches.

\section{References}

1. C.A. O'Flaherty, editor. Transport Planning and Traffic Engineering. Arnold, 1997. 544 p.

2. Sadoyoshi Mikami and Yukinori Kakazu. Self-organised control of traffic signals through genetic reinforcement learning. In Proceedings of IEEE Intelligent Vehicles, pages 113-118, 1993.

3. David J. Montana and Steven Czerwinski. Evolving control laws for a network of traffic signals. In John R. Koza, David E. Goldberg, David B. Fogel, and Rick L. Riolo, editors, Proceedings of the first annual conference on Genetic Programming, Stanford University, pages 333-338. A Bradford Book, July 1996.

4. Suh-Wen Chion. Optimisation of area traffic control subject to user equilibrium traffic assignment. In Proceedings of the 25th European Transport Forum. Transportation Planning Methods, volume 2, pages 53-64. Springer, 1995.

5. Carol Ashley. Traffic and Highway Engineering for Developments. Blackwell Scientific publications, 1994. 174 p.

6. Gordon Russell, Paul Shaw, and Neil Ferguson. Accurate rapid simulation of urban traffic using discrete modelling. Technical Report RR-96-1, Napier University, January 1996.

7. Gordon Russell, Paul Shaw, John McInnes, Neil Ferguson, and George Milne. The rapid simulation of urban traffic using field-programmable gate arrays. In Proceedings of the International Conference on the Application of New Technologies to Transport Systems. Australasian Road Research Board Ltd, May 1995.

8. Martin Bate, Alex Cowie, George Milne, and Gordon Russell. Process algebras and the rapid simulation of highly concurrent systems. In Proceedings of the 18th Australasian Computer Science Conference, February 1995.

9. R. J. Salter. Highway Traffic Analysis and Design. MacMillan Education, second edition, 1990. 